\title{
Dynamics Study and Analysis of Laser-Induced Transport of Nanoferrofluid in Water Using Fluorescein Isothiocyanate (FITC) as Fluorescence Marker
}

\author{
Mohammad E. Khosroshahi' ${ }^{1,2,3}$, Maral Asemani ${ }^{1}$ \\ ${ }^{1}$ Laser and Nanobiophotonics Laboratory, Biomaterial Group, Faculty of Biomedical Engineering, Amirkabir University of \\ Technology, Tehran, Iran \\ ${ }^{2}$ Department of Mechanical and Industrial Engineering, University of Toronto, Toronto, Canada \\ ${ }^{3}$ MIS-Electronics, Nanobiophotonics and Biomedical Research Lab., Richmond Hill, Canada \\ Email:khosrom@mie.utoronto.ca
}

How to cite this paper: Khosroshahi, M.E. and Asemani, M. (2017) Dynamics Study and Analysis of Laser-Induced Transport of Nanoferrofluid in Water Using Fluorescein Isothiocyanate (FITC) as Fluorescence Marker. Journal of Modern Physics, 8, 2219-2244.

https://doi.org/10.4236/jmp.2017.814137

Received: November 16, 2017

Accepted: December 26, 2017

Published: December 29, 2017

Copyright $\odot 2017$ by authors and Scientific Research Publishing Inc. This work is licensed under the Creative Commons Attribution International License (CC BY 4.0).

http://creativecommons.org/licenses/by/4.0/

\begin{abstract}
FITC-conjugated nanoferrofluid (FNFF) was synthesized and characterized to study the dynamic of laser-induced transport of NPs in water. The results confirmed a definite laser-induced enhanced velocity of NPs $\left(100 \mu \mathrm{m} \cdot \mathrm{s}^{-1}\right)$ almost twice as much the without laser (i.e. Brownian motion). The diffusion coefficients of $17 \times 10^{-6} \mathrm{~m}^{2} \cdot \mathrm{s}^{-1}$ and $55 \times 10^{-6} \mathrm{~m}^{2} \cdot \mathrm{s}^{-1}$ were found for the cases without and with laser action respectively. The act of laser when switched on after NPs had reached the steady state was very prominent. The laser-induced heat and power generated by NPs were calculated $0.2 \mu \mathrm{W} \cdot \mathrm{cm}^{-3}$ and 0.4 $\mathrm{pW} \cdot \mathrm{cm}^{-2}$ respectively. Our experiment condition was non-adiabatic and that the heat generated was diffused into the surrounding. We considered the Maxwell's criteria $(\mathrm{Kp} / \mathrm{Kw}<10)$ for FNFF thermal conductivity and found a value of $1.2 \mathrm{Wm}^{-1} \cdot \mathrm{K}^{-1}$. Based on the Brownian diffusion and DLVO theory, at earlier times where the NPs are more dispersed within the medium are displaced faster. However, at later stages they become less mobile as they are agglomerated. The mechanisms for the enhanced mobility and laser transport of NPs are thought to be due to e.m.w induced force (i.e. an oscillatory motion) and laser absorptive force (i.e., photothermophoresis). A beam divergence of about $5.24^{\circ}$ (or $91 \mathrm{mrad}$ ) was determined. A non-linear behaviour of laser beam was observed as a trajectory path within the water due to thermal heating hence causing the change of refractive index of medium and redistribution of NPs concentration.
\end{abstract}




\section{Keywords}

Magnetite Nanoparticles, Nanoferrofluid, Argon Laser, Dynamics Study, Visualization

\section{Introduction}

Heat transfer and fluid flow are of great interest in science and engineering, thus the experimental quantitative and qualitative observations are part of a comprehensive understanding of the processes. However, the low thermal conductivity of conventional heat transfer fluids such as water, oil and ethylene glycol prohibit achieving efficient and compact heat transfer devices. As a result, ferrofluids or magnetic fluids, which are stable colloidal solutions consisting of ferromagnetic particles dispersed in a carrier fluid are used to improve the heat dissipation problems due to their thermal and rheological properties [1]. In the absence of an external magnetic field, the magnetic moments are randomly distributed and the fluid shows no magnetization. However, under an applied magnetic field, the magnetic moments are aligned in the direction of field, hence producing a net magnetization moment similar to ferromagnetic material. It is this property which makes ferrofluids behaviour comparable to paramagnetic materials. This was followed by recent advances in nanotechnology leading to the development of nanofluids to further enhance the thermal conductivity. Basically, nanofluid is a multiphase nanomaterial such as metallic or oxide nanoparticles whose thermal conductivity is an order of magnitude or two higher than the base fluid in which they are suspended. A nanofluid consisting of ferromagnetic nanoparticles is referred to as nanoferrofluid (NFF). Magnetite, $\mathrm{Fe}_{3} \mathrm{O}_{4}$, is a common magnetic iron, which with proper surface coating they can be chemically stable and well dispersed with uniform size distribution. When the size of these nanoparticles becomes so small that their dimension can be considered as a single domain, they lack a hysteresis loop and a possess high field irreversibility, high saturation field and extra anisotropy contributions i.e., SPION [2] [3]. Since, magnetic nanoparticles (MNPs) obey the Coulomb's law and are easily controlled by an external magnetic field, thus they can be utilized for various industrial and biomedical applications such as photonic devices [4] [5], hyperthermia [6] [7] targeted drug delivery [8] [9] bioimaging [10] [11] and biomolecular sensing [12] [13].

Recently, there have been growing interests in utilizing electromagnetic waves such as laser to transport microparticles in liquids and remotely control matter due to its versatility and precision [14] [15]. Laser imaging is recognized as one of the most valuable diagnostic tool in fluid dynamics applications where instantaneous flow images can be measured with high spatial and temporal resolution. Optical techniques as versatile non-invasive tools such as speckle velocimetry [16] and Schlieren [17] have been playing a key role for achieving a better understanding by visualizing the fluid motion and temperature distributions in 
real time. In a research, Wataral et al. [18] used the combination of laser scattering force and a photothermal effect for electromagnetophoresis, $\mathrm{Pu}$ et al. [19] studied the laser-induced optical effects in magnetic fluid, Weinert et al. [20] showed how fluid can be moved by laser scanning microscope and Feng et al. [21] investigated enhanced mass transfer in nanofluids. Laser-induced fluorescence (LIF) is another versatile and practical laser imaging technique for variety of industrial applications such as measuring scalar flow parameters for example, concentration, mixture fraction, fluid composition [22] and biomedical applications for example, early cancer detection [23] and nanoparticle-enhanced imaging [24] and blood protein analysis [25]. As a conventional silanisation reagent, 3-amino-propyl-triethoxy-silane $\left[3 \mathrm{NH}_{2}\left(\mathrm{CH}_{2}\right) 3 \mathrm{Si}\left(\mathrm{OC}_{2} \mathrm{H}_{5}\right)\right.$-(APTES) can introduce amine groups onto the surface of the nanoparticle. In this way, one of the three ethoxy groups $\left(-\mathrm{O}-\mathrm{CH}_{2}-\mathrm{CH}_{2}-\mathrm{CH}_{3}\right)$ initially present in the APTES molecule breaks off from the molecule, which leads to covalent bonding between the APTES groups and the MNPs [10] At $\mathrm{pH}<10$, protonation of the amines may give a positive surface charge, which accounts for the high stability of these dispersions. The modification results in products with new functional groups on their surface, capable of interacting with an organic medium, and hence allowing the surface properties to change from typically hydrophilic silanol groups, which can be easily coupled with 3-aminopropyltriethoxysilane (APTES) to hydrophobic (lyophilic) ones. The active amino groups $\left(-\mathrm{NH}_{2}\right)$ facilitate the further functionalization and can covalently bond with other active groups, such as the carboxyl $(-\mathrm{COOH})$ that can conveniently conjugate with antibodies and other functional groups. Consequently, specific targeting and multifunctionalization can be realized. Additionally, the modified magnetic NPs with amino silane shell are non-toxic, biocompatible and injectable, which is highly important when considering biomedical applications. Fluorescein Isothiocyanate (FITC) is a derivative of fluorescein used in various applications such as flow cytometry and it is the original fluorescein molecule functionalized with an isothiocyanate reactive group $(-\mathrm{N}=\mathrm{C}=\mathrm{S})$, replacing a hydrogen atom on the bottom ring of the structure. FITC allows facile labelling of various structures and has been employed in laser-induced fluorescence detection techniques and flow cytometry of protein labelling [26] [27]. In our recent reports, it was shown that the laser-induced emission of FITC intensity can be used for cancer cells imaging [28] [29]. Following the previous works, we describe the possibility of application of FITC fluorochrome as LIF imaging agent to conjugate magnetic nanoparticles (FMNPs) and use it in the form of FITC-nanoferrofluid (FNFF) as optical marker to investigate the dynamic behaviour of FNFF in water base environment under influence of applied laser radiation.

\section{Materials and Methods}

\subsection{Materials}

All the chemicals including ferric chloride hexahydrate $\left(\mathrm{FeCl}_{3} \cdot 6 \mathrm{H}_{2} \mathrm{O}, 99 \%\right)$, ferrous chloride tetrahydrate $\left(\mathrm{FeCl}_{2} \cdot 4 \mathrm{H}_{2} \mathrm{O}, 99 \%\right)$ and hydrochloric acid $(\mathrm{HCl}$ 
$37 \%)$, ammonia aqueous (25 wt. \%), sodium hydroxide of $(\mathrm{NaOH}>99 \%)$, 3-amino-propyl-triethoxy-silane [3 $3 \mathrm{NH}_{2}\left(\mathrm{CH}_{2}\right) 3 \mathrm{Si}\left(\mathrm{OC}_{2} \mathrm{H}_{5}\right)$, APTES], Milli-Q water $(18.2 \mathrm{M} \Omega \cdot \mathrm{cm})$ were purchased from Merck and used without further purification and fluorescein isothiocyanate (FITC) was purchased from Aldrich Chemical Co. Dulbecco's Modified Eagle Medium (DMEM) culture medium was purchased from Sigma-Aldrich.

\subsection{Preparation of Magnetite Nanoparticles MNPs}

An amount of $1.28 \mathrm{M}$ ferric chloride hexahydrate $\left(\mathrm{FeCl}_{3} \cdot 6 \mathrm{H}_{2} \mathrm{O}, 99 \%\right), 0.64 \mathrm{M}$ ferrous chloride tetrahydrate $\left(\mathrm{FeCl}_{2} \cdot 4 \mathrm{H}_{2} \mathrm{O}, 99 \%\right)$ and $0.4 \mathrm{M}$ hydrochloric acid were prepared as iron source by dissolving the respective chemicals in $18.2 \mathrm{M} \Omega$ milli-Q water and deoxygenated by bubbling $\mathrm{N}_{2}$ gas for $1 \mathrm{~h}$ prior to the use under vigorous stirring at $25^{\circ} \mathrm{C}$. Similarly, $1.5 \mathrm{M}$ of $\mathrm{NaOH}$ was prepared as the alkali source. Flowing $\mathrm{N}_{2}$ gas not only protects the critical oxidation but also reduces the particle size when compared with methods without removing the oxygen. This is mainly because of generation of bubbles in the reaction solution due to the use of high stirring rates which may cause MNPs to be oxidised. A complete precipitation of $\mathrm{Fe}_{3} \mathrm{O}_{4}$ should be expected between $7.5-14 \mathrm{pH}$, while maintaining a molar ratio of $\mathrm{Fe}^{2+}: \mathrm{Fe}^{3+}=1: 2$ under a non-oxidising environment.

Aqueous dispersion of magnetic nanoparticles was prepared by alkalinizing an aqueous mixture of ferric and ferrous salts with $\mathrm{NaOH}$ at room temperature. 25 $\mathrm{mL}$ of iron source was added drop-wise into $250 \mathrm{~mL}$ of alkali source under constant magnetic stirring at $1500 \mathrm{rpm}$ for $30 \mathrm{~min}$ at ambient temperature. The precipitated powder was then isolated by applying an external magnetic field, and the supernatant was removed from the precipitate by decantation. The powder was washed and the solution was decanted twice after centrifugation at $5000 \mathrm{rpm}$ for $15 \mathrm{~min}$. Then $0.01 \mathrm{M} \mathrm{HCl}$ was added to neutralize the anionic charge on the particle surface.

\subsection{Amino-Silane Functionalization of MNP}

$25 \mathrm{~mL}$ of magnetite colloid ethanol solution was prepared, and then diluted to $150 \mathrm{~mL}$ by ethanol and $1 \mathrm{~mL} \mathrm{H}_{2} \mathrm{O}$. The solution was treated by ultrasonic wave for 30 minutes to uniformly disperse the particles and followed by adding $35 \mu \mathrm{L}$ of APTES to it and stirred for $7 \mathrm{~h}$. Amino groups are functionalized on the nanoparticle surface by silanization reaction. The feasibility of functionalization reaction could be confirmed visually by observing the precipitation of APTS functionalized magnetic NPs while remaining a clear ethanolic solution at the top. The solution was finally washed five times with ethanol and then dried into powder at room temperature under vacuum.

\subsection{FITC Conjugation of MNP-APTS}

This is done by adding $1 \mathrm{~mL}$ of $1 \mathrm{mg} / 1 \mathrm{~mL}$ of FITC molecules in Dimethyl sulfoxide $\left(\mathrm{CH}_{3}\right)_{2} \mathrm{SO}$, (DMSO) solution to $20 \mathrm{~mL}$ of $0.0128 \mathrm{M}$ of APTS functionalized 
iron oxide nanoparticles in ethanol. The conjugation was achieved between isothiocyanate functional group in FITC structure with high tendency to react with amine groups on $\mathrm{Fe}_{3} \mathrm{O}_{4}$ nanoparticles. The solution was placed on the shaker at low temperature for $4 \mathrm{~h}$ in dark room. The FITC conjugated MNPs were then magnetically separated from ethanol. To remove the unreacted FITC molecules, the MNPs were washed three times by ethanol and distilled water then dispersed in $20 \mathrm{~mL}$ of deionized water. The final product was centrifuged and kept in dark container. The schematic preparation of FMNP with an example of prepared sample is illustrated in Figure 1.

\section{Characterization}

Transmission electron microscope (TEM-Model CM120, PHILIPS) with NIH Image J software (http://rsb.info.nih.gov/ij/) was used to measure the sizes of MNPs. Fourier transformation-IR (FT-IR) spectra of samples were obtained using a FTIR spectrophotometer (NEXUS 670, Nicolet). X-ray diffraction was performed (FK60-40, $\lambda=1.5406^{\circ} \mathrm{A}, 40 \mathrm{kV}, 40 \mathrm{~mA}$ ) to determine the crystalline phase of NPs. Magnetization measurements were carried at $300 \mathrm{~K}$ in a magnetic field up to $8.5 \mathrm{k}$ Oe with a vibrating sample magnetometer (VSM-PAR 155). Zeta potential of MNP and FMNP along with hydrodynamic diameter of FMNP were determined through zeta sizer (Malvern, Nano ZS). The evaluation of fluorescence emission of FMNPs nanoparticles was performed using a $150 \mathrm{~mW}$ tunable ion argon laser Melles Griot-35MAP431 with wavelengths range between $(454-514) \mathrm{nm}$. The fluorescence signals were detected by a $600 \mu \mathrm{m}$ core diameter optical connected to spectrometer (UV-Vis USB 4000, Ocean Optics).

\section{Results}

TEM shown in Figure 2 clearly indicates the octahedral-like geometry of dispersed MNPs. The histogram covered a size range between $(8-20) \mathrm{nm}$ with an average distribution at $12 \mathrm{~nm}$ diameter. The effect of the chemical potential on

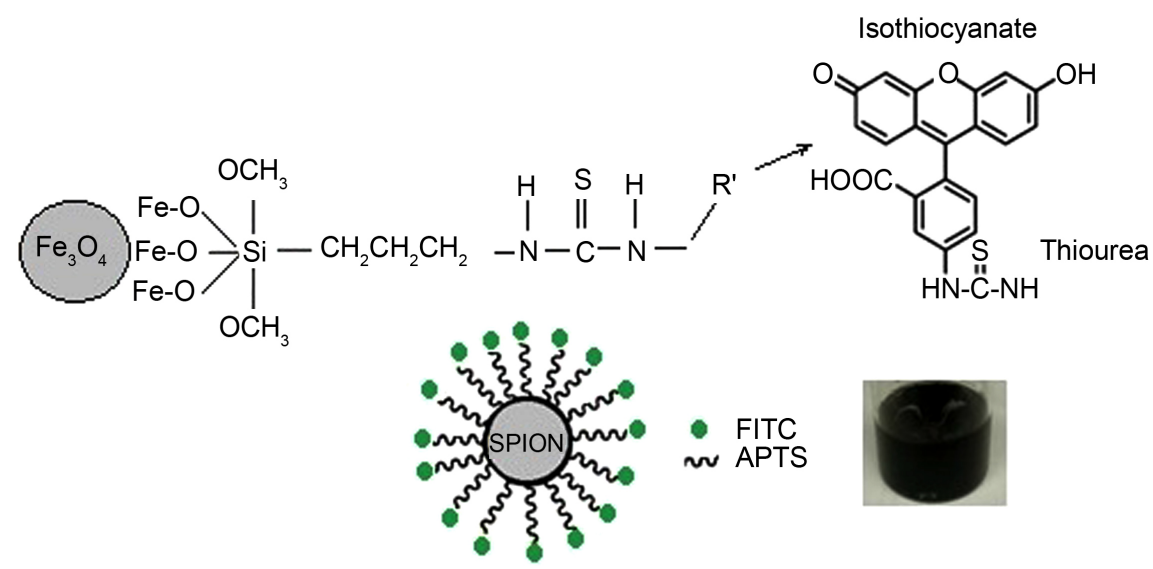

Figure 1. Schematic diagram of synthesis process of conjugating magnetite nanoparticle with FITC. 


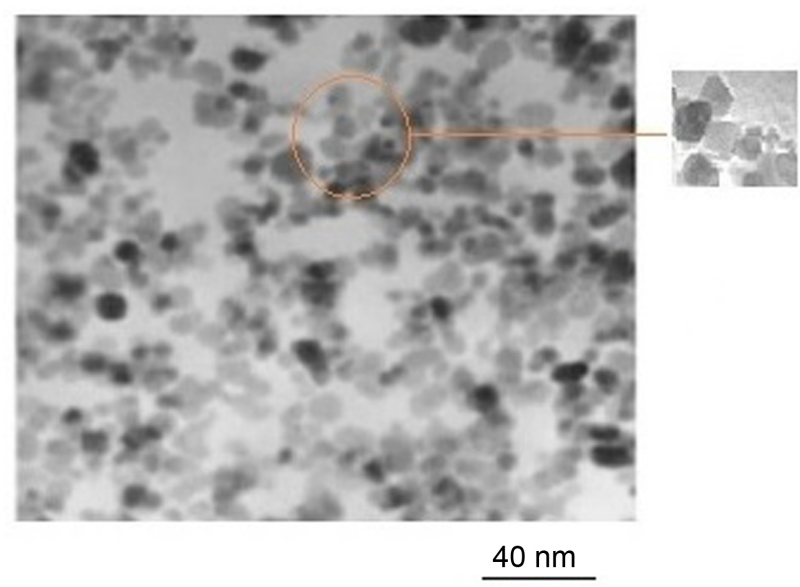

Figure 2. Transverse electron microscope images of MNPs.

the shape of crystals is discussed by Jin et al. [30]. In the case of crystal growth, it would be beneficial to have a higher chemical potential, which is mainly determined by the $\mathrm{NaOH}$ concentration. Octahedral $\mathrm{Fe}_{3} \mathrm{O}_{4}$ with high quality and crystallinity could be obtained in concentrated solution, because higher $\mathrm{OH}^{-}$ion concentration and higher chemical potential in the solution favor the growth of octahedral structures over other possible iron-oxide crystal forms.

The crystalline structural of $\mathrm{Fe}_{3} \mathrm{O}_{4}$ powders obtained under vigorous mechanical stirring were analyzed by XRD Figure 3(a). The results indicated no difference with pure MNPs and confirmed the formation of highly purified magnetite phase of iron oxide with diffraction peaks at (220), (311), (400), (422), (511), (440), which are the characteristic peaks of the $\mathrm{Fe}_{3} \mathrm{O}_{4}$ single-phased cubic inverse spinel structure (JCPDS file No. 19-0629) without any interference with other phases of $\mathrm{Fe}_{\mathrm{x}} \mathrm{O}_{\mathrm{y}}$. The inter-planar space (d-value) of synthesized nanoparticles can be calculated using Bragg equation for the reflection peaks. This can be used to distinguish between $\gamma-\mathrm{Fe}_{2} \mathrm{O}_{3}$ and $\mathrm{Fe}_{3} \mathrm{O}_{4}$ crystallographic structure. The broadening sources of the dominant intense peak in XRD graph such as 311 are preliminarily attributed to factors including ultra-fine particles, dislocations and grain boundaries. All the peaks in the patterns of the $\mathrm{Fe}_{3} \mathrm{O}_{4}$ NPs can be indexed with the cubic structure corresponding to magnetite phase. The average size of the crystals was estimated using Scherrer's formula,

$$
D_{h k l}=\frac{k \lambda}{\sqrt{B_{M}^{2}-B_{S}^{2}} \cos \theta_{h k l}}
$$

where $k$ is the shape factor, $\lambda$ the $\mathrm{X}$-ray wavelength, $B_{M}$ the half maximum line width (FWHM) in radians, $B_{S}$ the half maximum line width of the instrument, $\theta$ the Bragg angle and $D_{h k l}$ the mean size of the ordered (crystalline) domains. The dimensionless shape factor has a typical value of about 0.9 , but varies with the actual shape of the crystallite. The crystalline size of MNPs synthesized at $25^{\circ} \mathrm{C}$ was calculated as $18 \mathrm{~nm}$ using Debay-Sherrer's equation. To observe the agglomeration state, particle size distribution, polycrystalline electron diffraction 


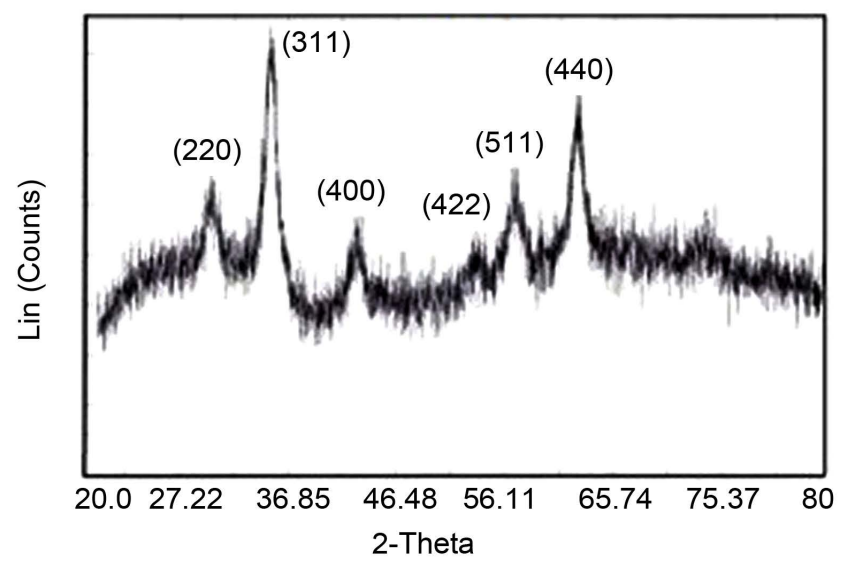

(a)

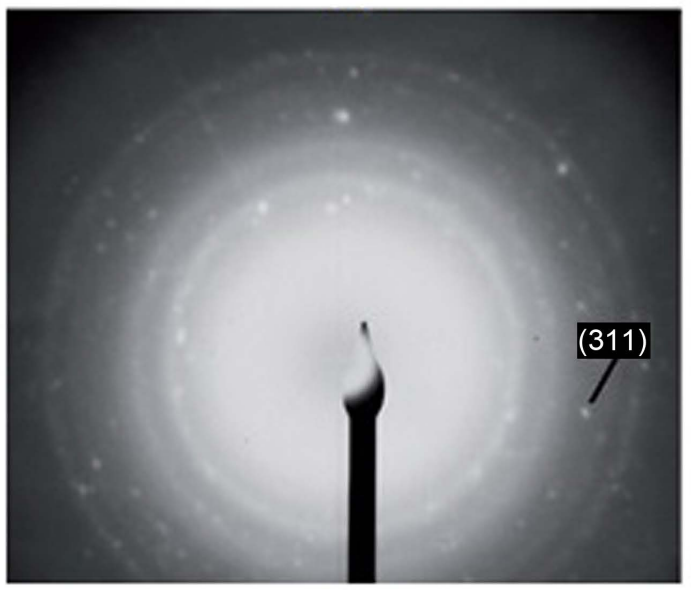

(b)

Figure 3. (a) X-ray diffraction pattern (XRD) of MNPs and (b) Polycrystalline electron diffraction pattern of the magnetite phase corresponding to MNPs.

pattern (EDP) was used, Figure 3(b) where the reflection corresponds to diffraction plane (311) characteristic of the magnetite phase.

To confirm the chemical bonding between APTES molecules and iron oxide nanoparticles and also between FITC molecules and amine functional group at MNP surface, FTIR spectroscopy was performed, Figure 4. Number of important determining factors for adsorption of aqueous solution ions on the surface of MNPs are high surface to volume atoms ratio, high nanoparticle surface energy and number of bonds at the surface. In a neutral solution containing dispersed MNPs, free $\mathrm{Fe}$ and $\mathrm{O}$ atoms at the surface adsorb $\mathrm{H}^{+}$and $\mathrm{OH}^{+}$which explains the high density of functional group $(-\mathrm{OH})$. The FTIR spectra of MNPs are shown in Figure 4(a) where the presence of magnetite, $\mathrm{Fe}-\mathrm{O}$, is evident at around $444 \mathrm{~cm}^{-1}$, $591 \mathrm{~cm}^{-1}$ and $1420 \mathrm{~cm}^{-1}$ and $1560 \mathrm{~cm}^{-1}$ and $3413 \mathrm{~cm}^{-1}$ show $-\mathrm{OH}$ bonds. The peaks related to $\mathrm{Fe}-\mathrm{O}$ bonds at amine group functionalized MNP surface are shifted to higher wave numbers of $477 \mathrm{~cm}^{-1}$ and $620 \mathrm{~cm}^{-1}$ respectively compared to pure $\mathrm{Fe}_{3} \mathrm{O}_{4} \mathrm{NPs}$, which confirms Fe-O-Si bond formation. Also, the $\mathrm{Fe}-\mathrm{O}-\mathrm{H}$ groups at $\mathrm{Fe}_{3} \mathrm{O}_{4}$ surface are replaced by $\mathrm{Fe}-\mathrm{O}-\mathrm{Si}(\mathrm{O}-)_{2}-\mathrm{R}$. 


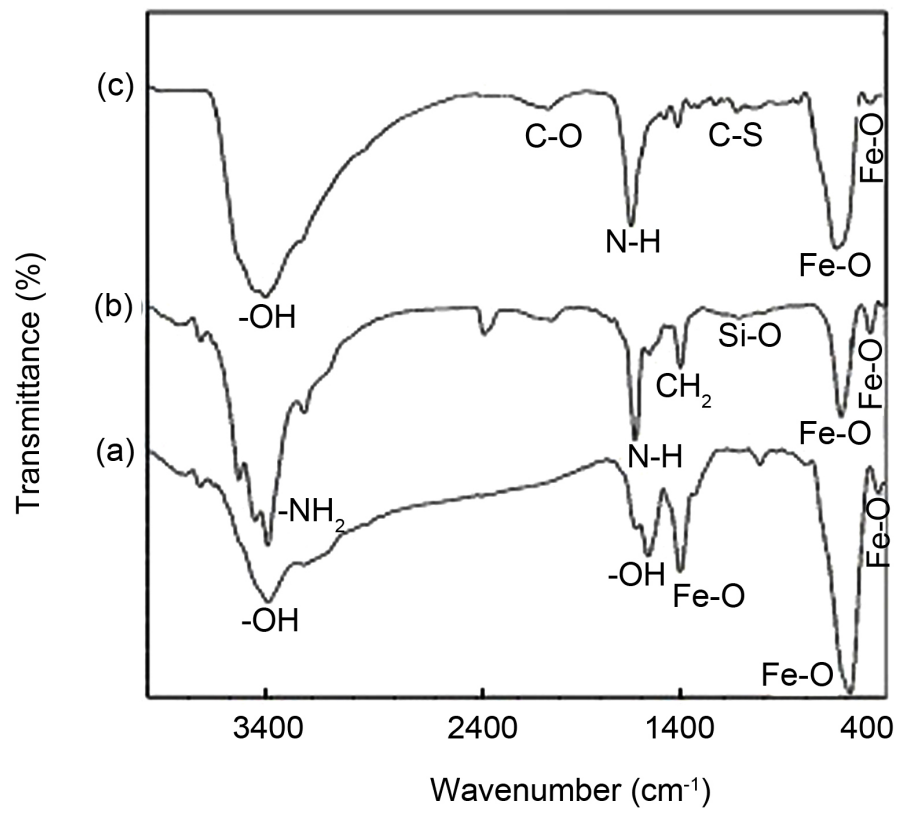

Figure 4. FTIR spectra of (a) MNPs, (b) APTS functionalized MNPs, (c) APTS functionalized MNPs conjugated by FITC.

Figure 4(b) indicates APTES functionalized MNPs spectrum where the 1118 $\mathrm{cm}^{-1}$ and $1403 \mathrm{~cm}^{-1}$ represent the stretching vibration of $\mathrm{Si}-\mathrm{O}$ and $\mathrm{CH}_{2}$ bands respectively and those near $1623 \mathrm{~cm}^{-1}$ and $3414 \mathrm{~cm}^{-1}$ the $\mathrm{NH}_{2}$. Figure 4 (c) represents the results of MNPs conjugated by FITC where the bonds at 1109 $\mathrm{cm}^{-1}, 1625 \mathrm{~cm}^{-1}, 2029 \mathrm{~cm}^{-1}$ and $3413 \mathrm{~cm}^{-1}$ correspond respectively to C-N stretched-vibrational, rotational-vibrational of amine, vibrational groups of $\mathrm{C}-\mathrm{O}$ present in FITC molecule structure and the vibrational group of $-\mathrm{OH}$ present in FITC molecule structure. It worth to noting that during the reaction between isothiocyanate group and amine, the functional group of (-NCS) in the FITC molecule structure is converted to thiourea (NH-CS-NH-) group, thus cannot be observed.

The VSM result in Figure 5(a) shows the room-temperature magnetization curve of the bare MNPs where the hysteresis loop exhibits a reversible behaviour implying the magnetization curve has zero remanence and Hc. The Ms value was measured $50 \mathrm{emug}^{-1}$ at $6000 \mathrm{Oe}$ and when the external magnetic field was removed, the particles redispersed rapidly. In some application such as switching the relatively fast magnetic response can be an advantage. In the case of $\mathrm{Fe}_{3} \mathrm{O}_{4}$ conjugated FITC, Figure 5(b), the Ms value decreased to about $40 \mathrm{emug}^{-1}$ indicating that FITC coating can lead to the formation of a nonmagnetic layer on top of the magnetic core which can consequently decrease the magnetization of the nanoparticles [31]. It is reported that Ms of iron-oxide nanoparticles increases with increase in particle size [32], which also may be due to different chemical compositions on the surface such as oxidation of $\mathrm{Fe}_{3} \mathrm{O}_{4}$ to $\mathrm{Fe}_{2} \mathrm{O}_{3}$, surface effect such as nonlinearity of spins of magnetically inactive layer with the magnetic field. Also, the discrepancy could be explained by the variation of 


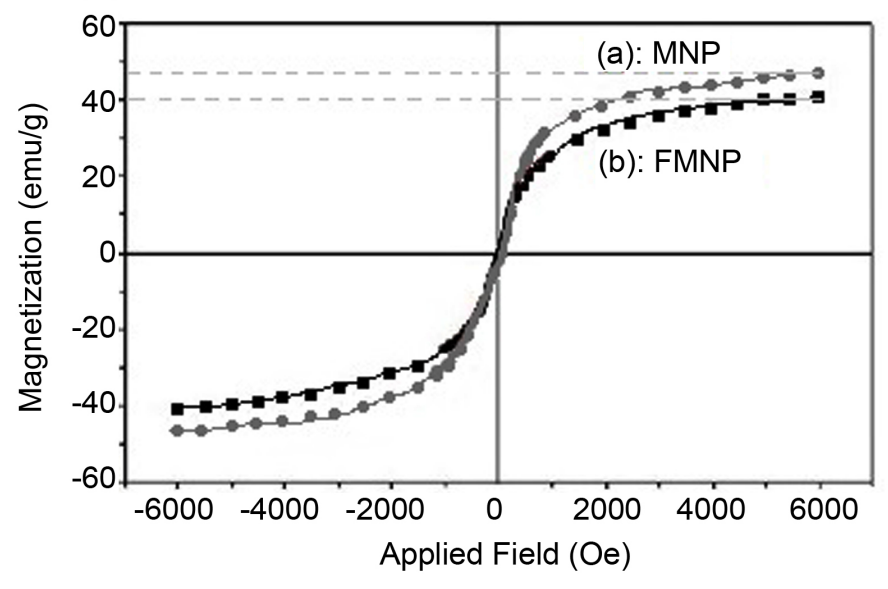

Figure 5. (a) Vibrating sample magnetometry (VSM) of MNPs and (b) FMNP.

synthesizing methods which can produce particles of different size.

In a separate experiment, the temperature of FNFF with nanoparticle concentration of $100 \mu \mathrm{g} / \mathrm{ml}$ in base water, irradiated by Ar laser at $488 \mathrm{~nm}$ for $5 \mathrm{~min}$. was measured. The temperature rise was measured using a digital K-type thermocouple thermometer (CHY502A1, CHY Fire-mate Co., Taiwan) with a probe diameter of $0.5 \mathrm{~mm}$ and a response time of $0.1 \mathrm{~s}$ which was placed parallel and 2 $\mathrm{mm}$ away from the laser beam. The results shown in Figure 6 indicates that the temperature increases linearly up to $1 \mathrm{C}$ within $30 \mathrm{~s}$ where there onwards the curve deviates and the temperature continuous to increase up to $3 \mathrm{C}$ in $300 \mathrm{~s}$. The non-linear behaviour can be due to number of reasons such as possible agglomeration of MNPs, which in turn the changes in geometrical shape and optothermal properties can affect the interaction process and also, the change of refractive index in the vicinity of irradiated area because of thermal gradient may paly a role.

The experimental setup for dynamics study is illustrated in Figure 7(a) where $50 \mu \mathrm{g} / \mathrm{mL}$ droplet of dispersed FMNPs (i.e., the solute) was first introduced to test tube containing distilled water as base medium. The diffusion process and the dynamics of laser-induced motion at different stages were studied by fast photography. The Time-resolved studies were done by a fast Camera (Panasonic Super Dynamic WV-CCD CP450) connected to an optical microscope (Prior-UK) for viewing. The output was then saved by a PC for further analysis. Two separate experiments were performed at this stage, one in the absence of laser irradiation and the other with unfocused beam of laser irradiating the solution for defined duration of time. In each experiment, new but the same amount of FMNPs and water were used. By studying the frame images, it was possible to visualize the concentration front and the overall profile of solute. Figure 7 (b) illustrates the result of laser-induced fluorescence of FMNPs excited at $488 \mathrm{~nm}$ with a typical emission peak at $518 \mathrm{~nm}$. The inset illustrates an example of FMNPs taken by fluorescence microscope (Zeiss Axioskop-Germany). 


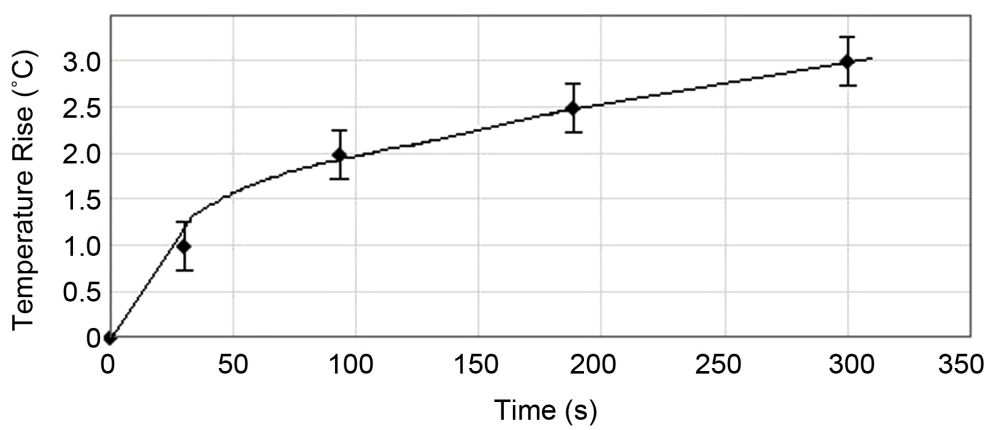

Figure 6. Temperature variation of FNFF in base water irradiated by Ar laser at $488 \mathrm{~nm}$ for $5 \mathrm{~min}$. The concentration of is $100 \mu \mathrm{g} / \mathrm{ml}$.

\section{SPION-FITC Colloids}

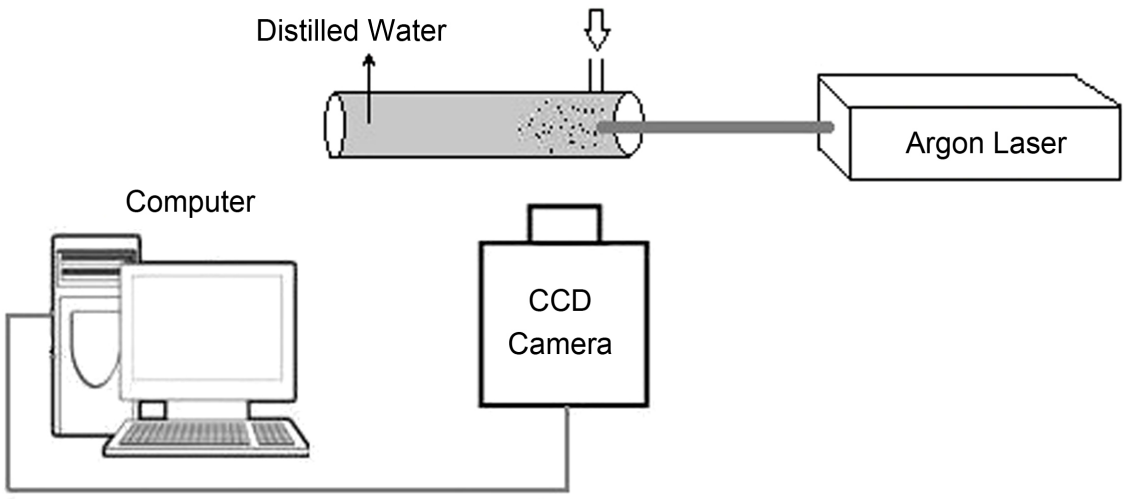

(a)

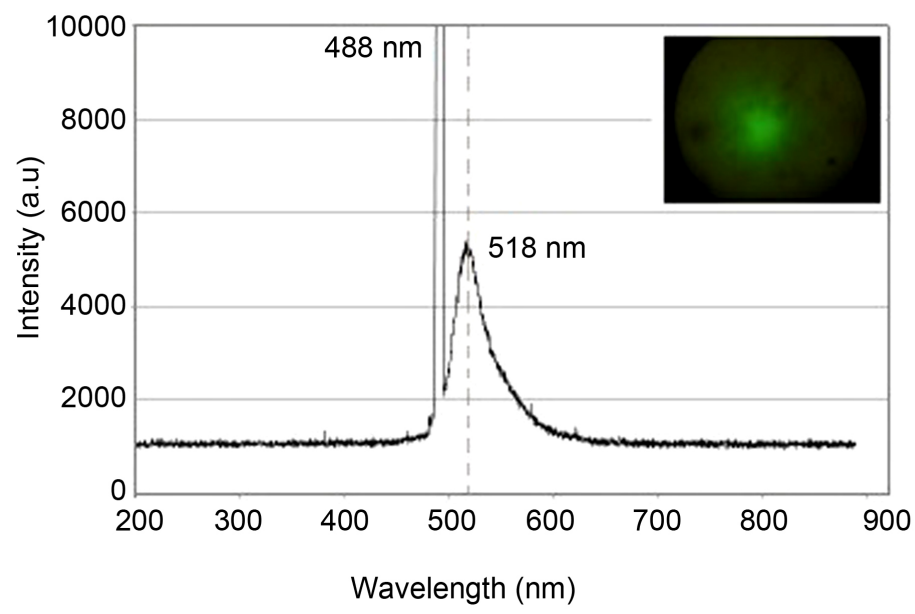

(b)

Figure 7. Experimental setup for studying the laser-induced FNFF motion.

Figure 8 represents the results of nanofluid diffusion due to Brownian motion at room temperature in the absence of laser radiation. The arrow indicates the diffusion front as a function of time, which in our case was recorded for $110 \mathrm{~s}$. According to micro convention model [33], the time required for a Brownian particle to travel its diameter $t_{B}$ is defined by 


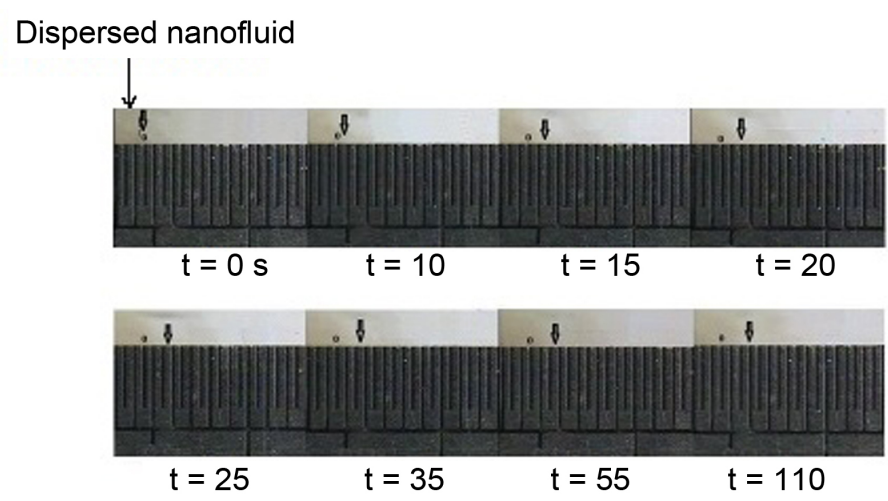

Figure 8. Time-resolved Brownian motion of FNFF in the absence of laser.

$$
t_{B}=3 \pi \eta D_{p}^{3} / 2 K_{B} T
$$

where $\eta=8.90 \times 10^{-4} \mathrm{~Pa} \cdot \mathrm{s}$ is the dynamic viscosity of base fluid (i.e., water in this case), $D_{p} \approx 12 \mathrm{~nm}$ is the nanoparticle diameter, $T \approx 300 \mathrm{~K}$ is the temperature and $K_{B}=1.38 \times 10^{-23} \mathrm{~J} \cdot \mathrm{K}^{-1}$ is the Boltzmann constant. Using the above values, it yields $t_{B} \approx 1.7 \mu$ s. When the laser was applied, Figure 9 , the induced fluorescence and the transmitted beam were clearly observed in dark room. The bright region represents the LIF of populated FNFF where Figure 9(a) is before injection of FNFF and Figure 9(b) is after the injection. The laser beam travels horizontally through the central part of the medium from left to right. The motion of fluid flow was recorded by video camera with a constant depth of field lens from a direction perpendicular to the optical axis. As it is seen in Figure 9(b), a random movement of medium was observed when the FNFF was poured into the glass tube. The FNFF movement was recorded from the initial injection time until it reached the steady-state condition. When the nanoparticles reached the beam, they were dragged inward to the beam and rapidly accelerated in the direction of the beam with very bright light scattering due to fluorescence. The sequence of time-resolved images is shown in Figure 9(c) with an example of magnification at $t=55 \mathrm{~s}$ (Figure 9(d)). It is interesting to note that as time elapses, the bright region or front also moves along the beam direction with slight but noticeable downward trajectory under influence of fluid flow, gravity and of some nanoparticles agglomeration due to dipole-dipole interaction mechanism as discussed in the next section.

The results of these experiments are shown in Figure 10 where it is clearly seen that in the absence of laser radiation the FNFF diffused a distance linearly up to $1.5 \mathrm{~mm}$ within $30 \mathrm{~s}$ where there onwards it reached the turning point and remained constant for rest of the experiment (i.e., $110 \mathrm{~s}$ ). The average velocity at constant rate corresponds to $\approx 50 \mu \mathrm{m} \cdot \mathrm{s}^{-1}$. However, in the second experiment when the laser was applied, FNFF travelled linearly up to $3 \mathrm{~mm}$ within the same time duration (i.e., $30 \mathrm{~s}$ ) but it continued further for another $1 \mathrm{~mm}$ within $75 \mathrm{~s}$. The average velocity in this case corresponds to $\approx 100 \mu \mathrm{m} \cdot \mathrm{s}^{-1}$, interestingly almost twice as much, which corresponds to an acceleration of $1.67 \mu \mathrm{m} \cdot \mathrm{s}^{-2}$. 


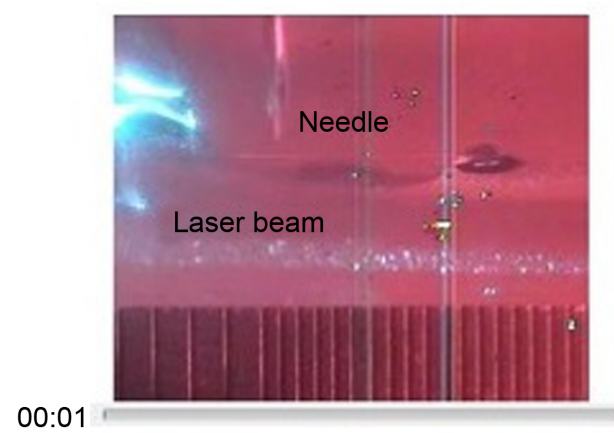

(a)

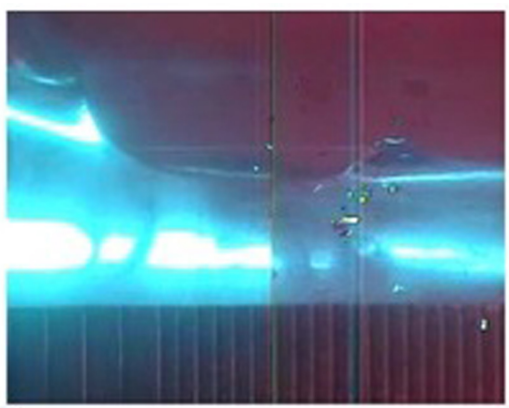

(b)

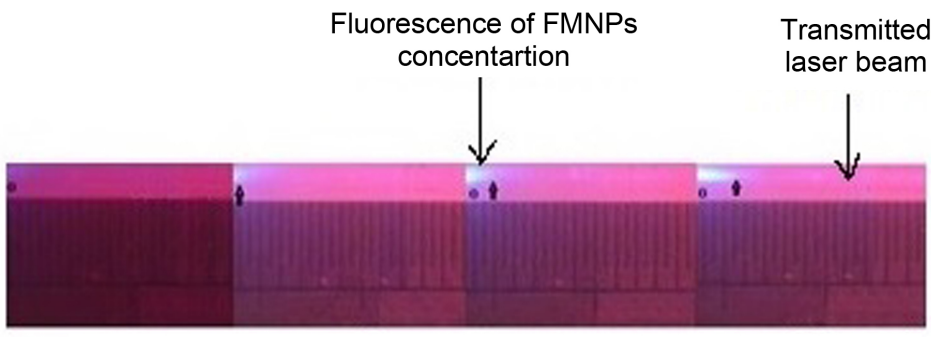

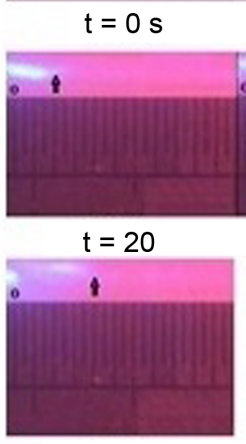

$t=5$

$t=10$

$t=15$

$t=110$

(c)

$$
\stackrel{\longmapsto}{4 \mathrm{~mm}}
$$

\section{(c)}

$06: 13$

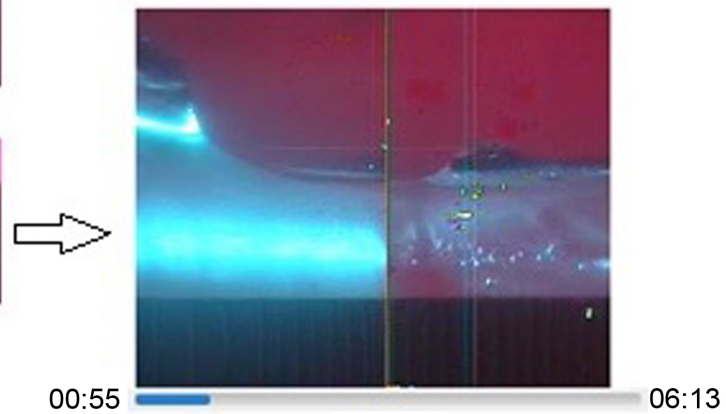

(d) $t=55$

$t=25$

$t=35$

00:55

Figure 9. Laser-induced motion of FNNF (a) before injection, (b) after the injection, (c) Time-resolved motion and (d) an example of LIF excitation of FNNF at $55 \mathrm{~s}$.

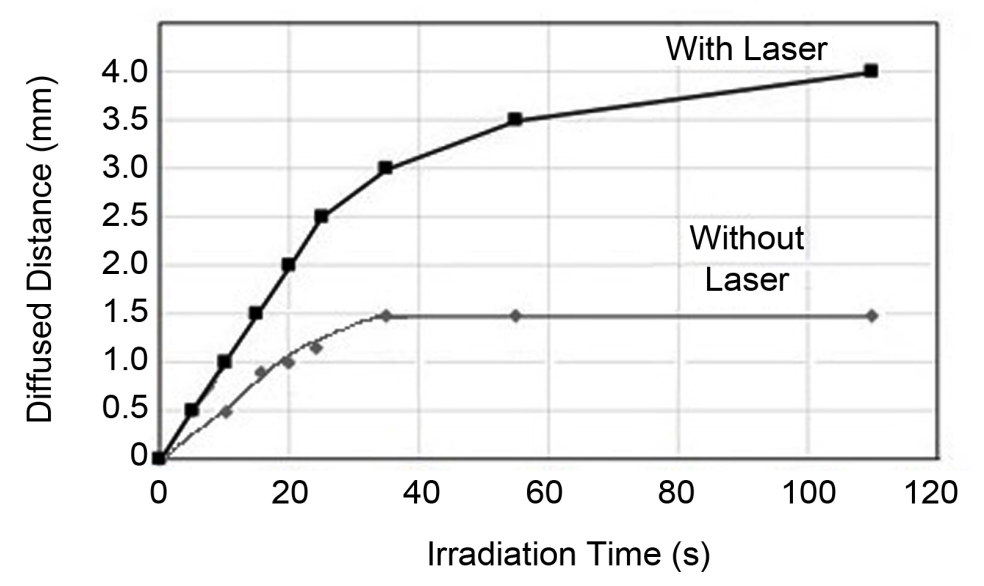

Figure 10. Measured diffused distance of FNFF without and with laser. Both curves almost reach the turning point after $25 \mathrm{~s}$ corresponding to 1 and $2.5 \mathrm{~mm}$ respectively. 


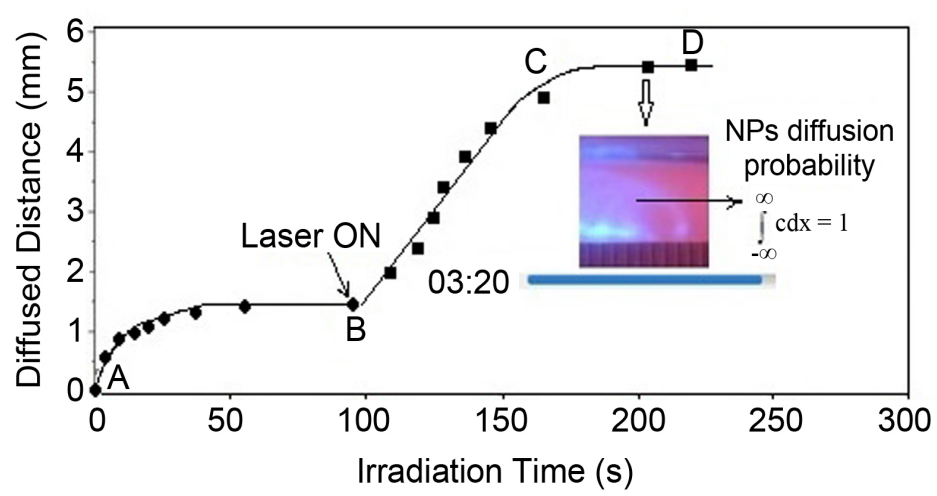

Figure 11. An independent experiment within the same water base. AB corresponds to the case without laser (i.e., Brownian motion) and $\mathrm{CD}$, the laser-induced. At point B when the laser was switched on, a rapid increase was observed, which saturates after about $170 \mathrm{~s}$. The inset indicates an example of FNFF distribution.

To test the effect of laser radiation on the nanoparticles, an independent experiment was performed, Figure 11, where the laser irradiated the sample in the same water medium after the FNFF had reached the steady-state i.e., the distance $\mathrm{AB}$ (similar to Figure 10). At this point, the laser was switched on (point $B$ ) and the motion was monitored again. A rapid initial linear increase in distance travelled by FNFF was observed up to $3.5 \mathrm{~mm}$ within $70 \mathrm{~s}$, which after wards they reached the plateau and remained almost constant at $5.5 \mathrm{~mm}$ in $225 \mathrm{~s}$ (i.e., the distance $\mathrm{CD}$ ). The inset indicates an example of FMNPs cloud faintly distributed around the main LIF optical axis excitation.

\section{Discussion}

\subsection{Absence of Laser Radiation}

It is known that the Brownian motion takes place because the particles so-called Brownian particles are under constant bombardment by the molecules of the surrounding liquid medium. These molecules exchange energy with the particles, but the direction of the velocity acquired by the particles is purely random and perhaps it is simplest way to treat the dynamics of nonequilibrium system. The irregular displacement of nanoparticles in nanofluid is mainly because of Brownian force and other forces between the fluid and particles such as friction, van der Waals and electrostatic. The interparticle forces depend on chemical and physical properties of NPs and base fluid, as well as their morphology and distribution. Such movements are the key factors for enhancement of heat and mass transfer of nanofluids. The fluctuation-dissipation theorem which relates the frictional and random forces to each other is expressed by Langevin equation.

$$
\frac{\mathrm{d} v(t)}{\mathrm{d} t}=-\frac{F_{f}}{m} v(t)+\frac{1}{m} \xi(t)
$$

where, $F_{f}=6 \pi \eta R_{p}$ (Stokes formula) is frictional force, $R_{p}$ is the radius of Brownian particle with mass $\mathrm{m}$ (i.e., FMNP) and $\xi(t)$ is a stochastic variable 
which gives the effect of background noise due to the fluid on the Brownian particle. To start the analysis, it is assumed that after $N$ collisions, the average distance $\langle x\rangle$ travelled by a particle between random collisions (its mean free path) is $(\langle N x\rangle)^{1 / 2}$ from its position at time $t=0$. Therefore, the distance travelled varies only with $t^{1 / 2}$ elapsed instead of being directly proportional to it. The net flow of particles from a region of higher concentration to one of lower concentration is defined by Fick's diffusion law,

$$
\frac{\partial c}{\partial t}=D \frac{\partial^{2} c}{\partial x^{2}}
$$

where $c$ is the concentration and

$$
D=\frac{K_{B} T}{F_{f}}
$$

is Fick's diffusion constant defined by Stokes-Einstein equation, that relates the diffusive flux to the concentration gradient of the particles. The intrinsic feature of 1-D Equation (4) is that the concentration is changing and consequently the concentration gradient is also changing during the diffusion process. Substituting the values, $R_{p}=6 \times 10^{-9} \mathrm{~m}$ and other values in Equation (5), it yields a value of $D \approx 4 \times 10^{-11} \mathrm{~m}^{2} \cdot \mathrm{s}^{-1}$. It is expected that in the absence of an external force, the velocity of NPs is weakly correlated over time and the surroundings drag on the NPs and hence they diffuse slowly. We may write a solution that satisfies the diffusion equation and the boundary conditions:

$$
c=\frac{1}{(4 \pi D t)^{1 / 2}} \mathrm{e}^{-x^{2} / 4 D t}
$$

The diffusion equation states that the speed at which the probability for finding the particle at $x(t)$ is position dependent. To normalize the concentration

$$
\int_{-\infty}^{\infty} c \mathrm{~d} x=1
$$

It follows that the value of $c$ at any $x$ and $t$ is the probability that a particle will have diffused to that position $x$ in the time $t$. Therefore, when the solute is injected, it begins to diffuse in the solution and in the absence of external force, the dispersed FMNPs undergo random Brownian motion. The 1-D diffusion process is governed by the mean square displacement, $\left\langle(\Delta x)^{2}\right\rangle$ equation,

$$
\left\langle(\Delta x)^{2}\right\rangle=2 D t
$$

where $t$ is the time taken for the mass displacement. In the absence of any isotropy in the diffusion medium, diffusion is perfectly described by the probability distribution of displacement's norm $x$ or $\left\langle(\Delta x)^{2}\right\rangle$. It is interesting to note that real displacements can be defined for any values of the time lag, whereas in the case of experimental observation, this number is limited to multiple of the frame duration $\Delta t$. Clearly, the amplitude of displacement decreases with time and the width of the curve spreads. One factor that influences directly on the velocity 
and the distance travelled by FMNPs is their agglomeration as explained below. The agglomeration process for colloidal particles results from the coupling between two main interactions: 1) particle-fluid interactions, which has a role in the motion of particles within a flow and govern the number of particle-particle encounters, and 2) particle-particle interactions, which determines if the colliding particles will adhere (adhesion or attractive interaction) or bounce (repulsive interaction). The latter process is described by the DLVO (Derjaguin, Landau, Verwey, and Overbeck) theory [34] [35] which defines inter-particle forces as the sum of van der Walls and double-layer electrostatic contributions. Based on this one can assume that the number of spherical solid particles $\left(N_{p}\right)$ dispersed in a medium (analogous to Gibbs energy) is proportional to the change of average particle diameter $\left(\left\langle D_{p}\right\rangle\right)$, equivalent to the coordination number at any time $(t)$

$$
N_{p}=Y\left(D_{p \max }-D_{p}\right)
$$

where $D_{p \max }$ is the maximum diameter that NPs can reach and $Y$ is a proportionality constant that considers the shape factor of the NPs. The variation of the number of NPs with respect to time due to agglomeration is

$$
-\mathrm{d} N_{p} / \mathrm{d} T=k_{g} N^{n}
$$

where $k_{g}$ is the agglomeration rate coefficient and $n$ is the reaction order. After some substitution and rearranging we get [36]

$$
d=d_{s}-\mathrm{e}^{\left(-k_{g} t\right)\left(d_{s}-1\right)}
$$

where $d=D_{p} / D_{p 0}, d_{s}=D_{p \max } / D_{p 0}$ and $D_{p 0}$ is the initial diameter (at $t=0$ $\left.D_{p}=D_{p 0}\right)$. Now, the attractive dipole interaction can be argued as follow. Many molecules are partially polar and have a net electric dipole moment, $\mu_{d}=Q \ell$. Here, $Q=q_{1} q_{2}$ is the electric charge and $\ell$ is the scalar distance. The vector electric field $\bar{E}$ produced by an electric dipole $\mu_{d}$ can be written

$$
\bar{E}=C^{\prime}\left[\frac{\mu_{d}}{r^{3}}-\frac{3\left(\mu_{d} \cdot r\right)}{r^{5}} r\right]
$$

This polar field resembles the magnetic field $B$ of a bar magnet, where $C^{\prime}$ is the coulomb constant $k=\left(4 \pi \varepsilon_{0}\right)^{-1}$, the overall strength of the dipole field is $\frac{C^{\prime} \mu_{d}}{r^{3}}$ and $r$ is the distance from dipole center to the field point. A second dipole $\mu_{d 2}$ will attract this field as $U=-\mu_{d 2} \cdot \bar{E}$. Thus, the interaction energy $U$ is about [37]

$$
U \cong-\frac{C^{\prime} \mu_{d 1} \mu_{d 2}}{r^{3}} \cong-\frac{C^{\prime} \ell_{0}^{2} e^{2}}{r^{3}}
$$

(Taking a value $\mu_{d}=e \ell_{0}$ ). The force associated with this interaction is $-\frac{\mathrm{d} U}{\mathrm{~d} r}$, this dipole-dipole force is attractive and the strength is

$$
F \cong-\frac{3 C_{2}^{\prime} \mu_{d 1} \mu_{d 2}}{r^{4}}
$$


Thus, the above arguments show that at early times where the NPs are likely more dispersed in the base medium will be displaced faster and freely and when they gradually become agglomerated they become less mobile with lower degree of freedom as the morphology of a fractal aggregate is characterized by its fractal dimension $\Phi_{f}$ which relates the mass of an aggregate $\left(M_{g}\right)$ to its radius of gyration $\left(R_{g}\right)$ as $M_{g} \propto R_{g}^{-\Phi_{f}}$. The diffusion of nanoparticle agglomeration, which depends upon their mass and morphology, plays an important role in the agglomeration kinetics and the final cluster size distribution.

Now using the experimental values of $\left\langle(x)^{2}\right\rangle$ and $t$ obtained from Figure 12 and substituting in Equation (8), it yields $D \approx 17 \times 10^{-6} \mathrm{~m}^{2} \cdot \mathrm{s}^{-1}$ and $\approx 55 \times 10^{-6} \mathrm{~m}^{2} \cdot \mathrm{s}^{-1}$ for the cases of without and with laser radiation respectively. As it is seen, these values, particularly without laser, are significantly larger than the theoretical value obtained from Equation (5). This may be partly due to error in observation and determining the diffusion front position, and secondly because of the effect of injection-induced perturbation force followed by the base medium microscopic fluctuation, which in turn can create secondary forced-oscillations hence enhanced displacement of nanoparticles. This causes so-called "localization uncertainty" which introduces a positive offset in $\left\langle(x)^{2}\right\rangle$. Another type offset as mentioned above is introduced by finite camera exposure. Sum of these effects can deviate the curve from linear to non-linear as observed in Figure 12. However, in the case of laser apart from the above reasons, it is expected to have a higher value of diffusion because of the interaction mechanisms. It is noteworthy that 1) there exists a time threshold for detection of nanofluid displacement in both cases corresponding to about 15 and $8 \mathrm{~s}$ respectively for without and with application of laser. This is considered as a limitation set by the measuring device as the displacement is expected to occur from the moment of injection, 2) regardless of the value of time thresholds, the fact remains

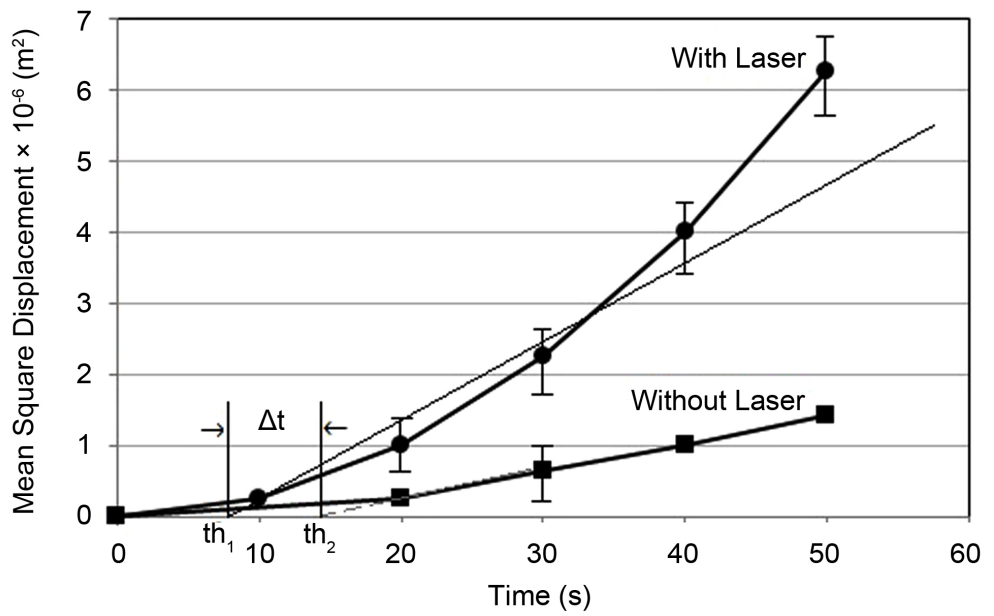

Figure 12. Measured mean square displacement of FNFF without and with laser. The thresholds indicate the minimum time for observing the displacement for without laser $(\approx 18 \mathrm{~s})$ and with laser $(\approx 8 \mathrm{~s})$ confirming the effect of laser radiation. 
that there is a difference between the two cases confirming the role of laser in accelerating the nanoparticles transfer, 3 ) as the time elapses, the divergence between the curves increases i.e., at later stages the divergence is enhanced by mechanisms such as thermal effect during the process.

\subsection{Laser-FNFF Interaction}

Generally, the interaction of light with particles depends strongly on number of parameters mainly the size, shape and composition of the particles as well as the medium in which the particles are embedded. A laser beam can irradiate nanofluid in a transparent liquid base either in the form pulsed or CW, where number of interaction forces can take place: 1) gradient where the laser beam is focused by a lens and the NP is trapped at the focal point by gradient force. This type of force tends to pull the NPs towards the center of the beam waist [10],2) direct where the scattering, reflection and refraction of photons creates a radiation force due to momentum transfer which tends to push the NPs [15], 3) indirect or radiometeric method where absorption of photons heats up the medium unevenly and creates a temperature rise gradient and NPs concentration is redistributed hence pushing them at different velocities, resulting in a force known as "photothermophoresis" [15] [38]. Upon the laser interaction, the NP will move in the direction of the laser propagation against the surrounding fluid during which, the fluid surrounding is expected to move hence creating microscale streamlines. In this case, we believe the mechanisms (2) and (3) play a key role simultaneously in transferring the MNPs.

\subsubsection{E.M.W-Induced Force}

Basically, the rationales behind the light interaction with metallic NPs are: electromagnetic wave (e.m.w) coupling, displacement of charges, dielectric polarization (i.e., electric dipole moment) and simple harmonic oscillation of the electric dipole moment of NPs excited by e.m.w. The induced oscillating field will then radiate a new wave which changes the distribution of the electromagnetic field followed by the changes in the momentum of NPs. Here we consider the interaction between a continuous wave (CW) Gaussian beam and nanofluid. For monochromatic planar waves, the characteristic solution of the Helmholtz equation is

$$
\left(\nabla^{2}+k^{2}\right) \underline{E}(r)=0
$$

In Cartesian coordinate, these waves are vectors waves with constant polarization vector $P(\bar{r})=E \chi \quad$ i.e., dipole moment/unit volume, initial amplitude $E_{0}$ and $\chi$ is the optical susceptibility.

$$
\underline{E}(r, t)=-R e\left[E_{0} P(\bar{r}) \mathrm{e}^{-i(k \omega-k r)}\right]
$$

where $\omega$ is the angular frequency of photon traveling in the z-direction has

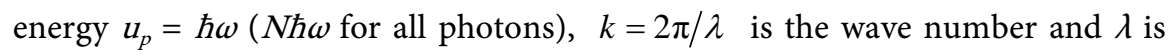
the laser wavelength. we define the wave vector by $k . r=$ constant where $r$ is the 
distance and planes with phase $\Phi=\omega t-k r$. Let us assume the total energy of an electromagnetic field $\left(u_{T}=u_{E}+u_{B}\right)$ is obtained by integration over the corresponding volume $(V)$ of spherical NPs,

$$
u_{T}=\varepsilon_{0} \int_{V}|\underline{E}(r)|^{2} \mathrm{~d}^{3} r
$$

where

$$
\begin{gathered}
u_{T}=u_{E}+u_{B} \\
u_{E}=\frac{1}{2} n^{2} \varepsilon_{0} E^{2} \text { and } u_{B}=\frac{1}{2 \mu_{0}} B^{2}
\end{gathered}
$$

$\varepsilon_{0}=8.85 \times 10^{-12} \mathrm{~F} \cdot \mathrm{m}^{-1}$ is the vacuum dielectric constant and permittivity and $\mu_{0}=4 \pi \times 10^{-7} \mathrm{H} \cdot \mathrm{m}^{-1}$ is the permeability of free space. The average field strength is

$$
\langle|\underline{E}|\rangle=\sqrt{N \hbar \omega / \varepsilon_{0} V}
$$

The Equation (18) is important if one wants to describe the coupling of the field oscillation to NP. But the energy of e.m.w is divided equally between its constituent $E$ and $B$ fields. The total energy density is then $u_{T}=E^{2} \varepsilon_{0}=\left(1 / \mu_{0}\right) B^{2}$. The rate of energy transported per unit area after the field coupling is described by time varying Poynting vector at the position where the NP is.

$$
\langle|S|\rangle=\varepsilon_{0} c^{2}\left\langle E_{0} B_{0} \sin ^{2}(k \cdot r \pm \omega t)\right\rangle=1 / 2\left(\frac{c}{\mu_{0}}\right) B_{0}^{2}
$$

Thus, the NP will experience a force given by [39]

$$
F_{P}=n_{m} / c \cdot\langle S\rangle \cdot \sigma_{x}
$$

where $c$ is the velocity of light, $n_{m}$ is the refractive index of surrounding medium and $\sigma_{x}=\sigma_{\alpha}+\sigma_{\beta}$ is sum of MNP absorption and scattering cross sections. In conducting materials charges can move freely but under influence of an applied external time varying electric field such as laser and to accelerate electrically charged NPs, which in doing so it generates polarization and current through displacement of charges. Thus, by using Maxwell's equation for time varying fields, we get

$$
\nabla \times \underline{H}=j+\frac{\partial}{\partial t} D_{d}
$$

where $D_{d}$ is dielectric displacement given by $\frac{\partial}{\partial t} D_{d}=j_{d i s}+j_{p o l}$ with $j_{d i s}$ and $j_{p o l}$ are displacement and polarization currents respectively. An important quantity in a metal dielectric response is the plasmon frequency defined as

$$
\omega_{p}^{2}=\frac{n_{e} e^{2}}{m_{e f f} \varepsilon_{0}}
$$

where $n_{e}$ is the number of electrons, $e$ is the electron charge $\left(1.6 \times 10^{-19} \mathrm{C}\right), m_{\text {eff }}$ is effective mass. We know that the dimensions of metallic NPs are so small that 
light can easily penetrate the whole NP and grasp at all conduction band electrons. The result is that the sea of conduction band electrons is displaced with respect to positively charged ions from the metallic lattice. The resulting electric dipole on the NP represents a storing force and hence the NP can be considered as harmonic oscillator, driven by a light wave and damped by some ohmic losses e.g. heat or as radiative (scattering) losses. The latter is equivalent to the re-emission of photon on the expense of NP plasmon (NPP) excitation.

In his model, electrons are considered that are harmonically bound to an ionic core with a spring (i.e. oscillatory atomic bond) and oscillating at optical frequencies $\omega_{0}$. The restoring force, $F_{r}=-m \omega_{0}^{2} x$ and by assuming that damping of the oscillator is caused by release of the radiation energy, the damping force is given by $F_{d}=-m \gamma\left(\frac{\mathrm{d} x}{\mathrm{~d} t}\right)$ where $\gamma$ is the damping rate and $\gamma \ll \omega_{0}$.

Assuming the complex trail function $r(t)=x+i y=\rho(t) \mathrm{e}^{-i \omega t}$, where, $E \cdot \mathrm{e}^{-i \omega t}$ is the driving light field and at equilibrium solution $\rho(t)=\rho_{0}=$ constant with

$$
\rho_{0}=\frac{q E / m}{\left(\omega_{0}^{2}-\omega^{2}\right)-i \omega \gamma}
$$

The "dispersive" $(x)$ and "absorptive" $(y)$ components of the radiation interaction can be written as

$$
r(t)=x+i y=\rho_{\max } \frac{\gamma}{2} \frac{\delta-i \gamma / 2}{\delta^{2}+(\gamma / 2)^{2}} \mathrm{e}^{-i \omega t}
$$

Therefore, the Equations (20) and (24) shows that NPs can be transported under direct influence of e.m.w oscillating field-induced force and in doing so the released energy of re-emitted dipole radiation can be dispersive and absorptive within the medium.

\subsubsection{Absorptive Force and the Effects}

1) Heat generation

The absorptive force and its effects within NFF can be discussed in terms of following factors: a) heat generation, b) temperature distribution, c) Thermal gradient and change of refractive index, d) thermal conductivity and divergence and e-beam trajectory. The heat generation involves both absorption of incident photons and the conversion of photons into heat energy as well as heat transfer from MNPs to the surrounding medium. In this case, it is assumed that the absorption cross section dominates the scattering cross section, $\sigma_{\alpha} \gg \sigma_{\beta}$. This is because the scattering is caused by the inhomogeneity in the refractive index of a medium and the spatial distribution of the scattering in turn depends on the size and shape of the inhomogeneity relative to the source wavelength. Here, the radius of NP, $R_{p} \approx 6 \mathrm{~nm} \ll \lambda / 20 \approx 24 \mathrm{~nm}$ and $x=2 \pi R_{p} / \lambda \approx 0.08<1$, thus, the light scattering can be described by Rayleigh theory when the anisotropy factor or the mean cosine of the scattering, $g=0$. However, when the NP size increases due to reasons explained above, the Mie scattering gradually dominates and the intensity distribution increases in the forward direction, $g=1$, and the scattering 
phase function $p\left(\hat{a}, \hat{s}^{\prime}\right)$ for small angles becomes much higher than for all other angles. $p\left(\hat{s}, \hat{s}^{\prime}\right)$ describes the fraction of light energy incident on the scatterer from $s$ direction that gets scattered in the new direction $s$. While scattering depends on the dimension of the NPs, the optical absorption depends on the type of material i.e., the chemical composition. Assuming $\alpha_{p}$ is the absorption coefficient $\left(\mathrm{cm}^{-1}\right)$ of MNP and $I_{0}$ is the laser initial intensity $\left(\mathrm{W} \cdot \mathrm{cm}^{-2}\right)$ and since $T(r, t)=\alpha_{p} \int_{0}^{t} \frac{I \mathrm{~d} t}{\rho_{p} c_{p}}$ we can write

$$
\frac{\partial T}{\partial r}=\frac{\alpha_{p}}{\rho_{p} c_{p}} \frac{\partial \int_{0}^{t} I \mathrm{~d} t}{\partial r}=\frac{\partial W}{\partial r}
$$

where $\rho_{p}$ and $c_{p}$ are the density and heat capacity of NP respectively, $\sigma$ is the induced pressure and $W$ is the amount of absorbed energy per unit volume. Using the Newton's second law of motion, we obtain

$$
\frac{\partial^{2} x}{\partial t^{2}}=\frac{1}{\rho_{p}} \frac{\partial \sigma}{\partial r}
$$

Thus, it can be seen that the rate of change of temperature is directly related to the absorption coefficient of material and the laser intensity, which eventually causes the external force to act upon the NPs. The complex refractive index, $\tilde{n}(\omega)$ of $\mathrm{Fe}_{3} \mathrm{O}_{4}$ nanoparticle is related to the NP complex dielectric permittivity $\varepsilon_{p}=\varepsilon_{r}+i \varepsilon_{i}$ through $\varepsilon_{p}=\tilde{n}^{2}$. The real part, $\varepsilon_{r}=n_{p}^{2}-k_{e}^{2}$, where $n_{p}$ is the real refractive index of NP, $k_{e}(\lambda)=\lambda \alpha_{p} / 4 \pi$ is extinction coefficient which determines the degree to which the metal polarizes in response to an applied external electric field, that is, it determines the SPR spectral peak position and is maximum when $\varepsilon_{p}+2 \varepsilon_{w}=0$ where $\varepsilon_{p}$ and $\varepsilon_{w}$ are permittivity of NP and water respectively and $\alpha$ is absorption coefficient of NP. The imaginary part, $i \varepsilon_{i}=2 n_{p} k_{e}$ however, quantifies the relative phase shift of this induced polarization with respect to the external field, i.e., it determines the bandwidth and includes losses such as ohmic heat loss. Taking $n_{p}($ at $488 \mathrm{~nm}) \approx 1.41$ [40] and $k_{e} \approx 1$ [41] then $\varepsilon_{r} \approx 0.98$ and $\varepsilon_{i} \approx 2.82$ and thus $\varepsilon_{p} \approx 3.8$ and $\varepsilon_{w} \approx 1.9$. In our case, radius of MNP is much smaller than optical penetration, $R_{p} \approx 6 \mathrm{~nm} \ll \delta_{o} \approx \alpha^{-1} \approx 38 \mathrm{~nm}$ and according to Mie theory, the absorption cross section, $\sigma_{\alpha}$ of a particle embedded in a medium, $\varepsilon_{m} \approx-\varepsilon_{p} / 2$, is given by [42]

$$
\begin{gathered}
\sigma_{\alpha}=\frac{8 \pi^{2}}{\lambda} R_{p}^{3}\left[\frac{\varepsilon_{p}(\omega)-\varepsilon_{w}}{\varepsilon_{p}(\omega)-2 \varepsilon_{w}}\right]^{2} \\
\eta_{\alpha}=\frac{\sigma_{\alpha}}{\pi R_{p}^{2}} \approx 1.9 \times 10^{-2}
\end{gathered}
$$

Now substituting the above values in Equations (27) and (28), yields $\sigma_{\alpha} \approx 2.18 \times 10^{-18} \mathrm{~m}^{2}$ and $\eta_{\alpha} \approx 1.9 \times 10^{-2}$. Since, $x \approx 0.08<1$, it is assumed that each MNP is quasi-transparent to the incident light. By taking the experimental values of laser power $P \approx 150 \mathrm{~mW}$, spot area $A \approx 7.83 \times 10^{-3} \mathrm{~cm}^{2}$ and $\alpha \approx 2.6 \times 10^{-5} \mathrm{~cm}^{-1}$ then the heat produced per unit volume $Q=I_{0} \alpha \approx$ 
$0.2 \mu \mathrm{W} \cdot \mathrm{cm}^{-3}$. Similarly, the heat power generated is $P=V_{p} Q=I_{0} \sigma_{\alpha} \approx$ $0.4 \times 10^{-12} \mathrm{~W}$ where $V_{p}$ is the NP volume. To calculate the heat generated inside a NP, it is assumed that the size of a MNP is smaller than the laser wavelength so that electrons inside the MNPs respond collectively to the applied electric field of the laser radiation $E_{0}\left[\frac{3 \varepsilon_{w}}{\varepsilon_{p}+2 \varepsilon_{w}}\right]$. The heat source is derived from the heat power density $h_{\rho}(r)=\int_{v} h_{\rho}(r) \mathrm{d}^{3} r$, where the integral is over $V_{p^{\text {. }}}$ (i.e. total heat generated $Q_{T}=V_{p} Q$ ).

\section{2) Temperature distribution}

When a laser beam with a Gaussian profile, intensity $I_{0}(r, t)$ and beam diameter $2 \mathrm{a}$ interacts with the NFF in water, the radiation is absorbed by the sample (i.e., $\alpha \gg \beta$ ) and subsequent nonradiative decay of excited MNPs electrons results in local heating of the medium. Secondly, $I_{0}(r, t)$ is exponentially attenuated at a radial distance $r$ within the medium and in the propagation direction (depth) $z$. described by Equation (27)

$$
I=1 / 2 c \varepsilon_{w}\left|E_{0}\right|^{2}\left|\frac{3 \varepsilon_{w}}{\varepsilon_{p}+2 \varepsilon_{w}}\right|^{2} \cdot \operatorname{Im}\left(\frac{\varepsilon_{p}-\varepsilon_{w}}{\varepsilon_{p}+2 \varepsilon_{w}}\right) \mathrm{e}^{-2 r^{2} / a^{2}} \cdot \mathrm{e}^{-\alpha z}
$$

The temperature distribution in the medium resembles the profile of the excitation beam and hence a refractive index gradient is created. The temperature distribution around the MNPs (i.e., heat source) placed in a surrounding medium (i.e., water) is described by the parabolic Fourier's heat conduction equation [43]

$$
\rho_{p}(r) c_{p}(r) \frac{\partial T(r, t)}{\partial t}=K_{w} \nabla^{2} T(r, t)+Q_{s}(r, t)
$$

where $T(r, t)(\mathrm{K})$ is local temperature,

$Q s=P / V_{p}=\frac{\omega}{8 \pi}\left|E_{0}\right|^{2}\left|\frac{3 \varepsilon_{w}}{\varepsilon_{p}+2 \varepsilon_{w}}\right|^{2} \cdot \operatorname{Im}\left(\frac{\varepsilon_{p}-\varepsilon_{w}}{\varepsilon_{p}+2 \varepsilon_{w}}\right)\left(\mathrm{W} / \mathrm{m}^{3}\right)$ is the heating source, $P$ is the power of heat generation (W), $\rho_{p} \approx 5240 \mathrm{~kg} / \mathrm{m}^{3}$, and $c_{p} \approx 640 \mathrm{~J} / \mathrm{kg} \cdot \mathrm{K}$ are density, and specific heat of $\mathrm{Fe}_{3} \mathrm{O}_{4}$, respectively, $K_{w} \approx 0.6 \mathrm{~W} \cdot \mathrm{m}^{-1} \cdot \mathrm{K}^{-1}$ is the thermal conductivity of the water and $\mathrm{r}$ is the radial distance from the heated nanoparticles. A characteristic time $t_{\mathcal{o}}$ to establish the temperature profile around a single $\mathrm{NP}$ is $\delta_{0}^{2} / 4 D_{\mathrm{t}}$ where $\delta_{0} \approx \alpha^{-1} \approx 38 \mathrm{~nm}$ and $D_{t} \approx 1.4 \times 10^{-7} \mathrm{~m}^{2} \cdot \mathrm{s}^{-1}$ are optical penetration depth and thermal diffusivity of water respectively, so $t_{c} \approx 2.6 \mathrm{~ns}$, which clearly is a very fast time. Thus, one can determine the thermal diffusion depth into MNP by substituting the value of $\left(D_{t}\right)_{p}=K_{p} / \rho_{p} c_{p} \approx 1.8 \times 10^{-6} \mathrm{~m}^{-2} \cdot \mathrm{s}^{-1}$ in $X_{T}=\left[4\left(D_{t}\right)_{p} \tau\right]^{1 / 2} \approx 2.6 \mathrm{~mm}$ for an exposure time $\tau=1 \mathrm{~s}$. Therefore, the condition $R_{p} \ll \delta_{0} \ll X_{T}$ or $t \gg t_{c}$ applies in our case i.e., a non-adiabatic case. In the steady-state regime, the local temperature around a NP (i.e., $r \geq R_{p}$ ) is described by [44]

$$
\Delta T(r)=\frac{P}{4 \pi K_{w}}=\frac{V_{p} Q}{4 \pi K_{w} r}=\frac{\int I \mathrm{~d} A_{p}}{4 \pi K_{w} r}=\frac{|I| A_{p}}{4 \pi K_{w} r}
$$


where $A_{p}$ is the area of NP and the according to Equation (27) the intensity decreases exponentially both in $r$ and $z$ directions. The temperature increases at the surface of NP (i.e., at $r=R_{p}$ ) is

$$
\begin{gathered}
\Delta T(r)=\frac{R_{p}^{2} K_{p}}{K_{w}} \cdot \frac{1}{2} c \varepsilon_{w}\left|E_{0}\right|^{2}\left|\frac{3 \varepsilon_{w}}{\varepsilon_{p}+2 \varepsilon_{w}}\right|^{2} \cdot \operatorname{Im}\left(\frac{\varepsilon_{p}-\varepsilon_{w}}{\varepsilon_{p}+2 \varepsilon_{w}}\right) \\
\Delta T(r)=\frac{I R_{p}^{2} K_{p}}{K_{w}} \cdot \operatorname{Im}\left(\frac{\varepsilon_{p}-\varepsilon_{w}}{\varepsilon_{p}+2 \varepsilon_{w}}\right)
\end{gathered}
$$

where $K_{p}$ is the thermal conductivity of MNP So, $\Delta T(r) \propto R_{p}^{2}$ and the total heat current from the surface of NP is given by $K_{w} A_{p} \partial \Delta T / \partial r$. It is interesting to note that the size dependence of the temperature increase is governed by the total rate of heat produced and by the heat transfer through the NP. Based on this fact, the temperature increases at later times observed in Figure 6 can be explained caused by for example the agglomeration effect.

\section{3) Thermal conductivity}

Since Maxwell's equation of thermal conductivity is only for first-order approximation, it applies only for mixtures with low particle volume fraction $V_{f}$ and small values of $K_{p} / K_{w}<10$, which in this case is $\approx 0.1$, so we can write [45]

$$
K_{m}=K_{w}\left[1+V_{f}\left(\frac{K_{p}}{K_{w}}\right)-1\right]
$$

Though the $K$ value of NFF depends on factors such as volume fraction, NP size, morphology, additives, $\mathrm{pH}$, temperature, base fluid and NP material [46] [47]. Here, $K_{p}=0.6 \mathrm{~W} \cdot \mathrm{m}^{-1} \cdot \mathrm{K}^{-1}, K_{w}=6 \mathrm{~W} \cdot \mathrm{m}^{-1} \cdot \mathrm{K}^{-1}$ and the volume fraction $V_{f}=0.012$ (for $100 \mu \mathrm{L} \mathrm{MNP} \mathrm{solution).} \mathrm{Using} \mathrm{the} \mathrm{above} \mathrm{values} \mathrm{in} \mathrm{Equation}$ (34) it gives $K_{m}=1.18 \mathrm{~W} \cdot \mathrm{m}^{-1} \cdot \mathrm{K}^{-1}$ at $T=300 \mathrm{~K}$. Therefore, on would expect a higher thermal conductivity by using smaller MNPs.

\section{4) Change of refractive index and beam trajectory path}

The heating can produce thermal gradient within the medium due to absorption of light energy and redistribute the concentration of MNPs. These factors can change the refractive index of NFF.

$$
\frac{\partial[\Delta T(r, z)]}{\partial r}=\frac{B a^{2}}{4 K_{p}} \frac{1}{r}\left(\mathrm{e}^{-2 r^{2} / a^{2}}-1\right) \mathrm{e}^{-\alpha z}
$$

where $B=\alpha P / \pi a^{2}$ and $P$ is the laser power, $a$ is the laser beam radius. Therefore, thermal and concentration diffusion of MNPs occur due to local heating by the laser beam inside the NFF [15] [21] [48]. It is noteworthy that $\Delta n$ can be caused by both thermal and nonthermal effects where in the first case, the change of refractive index caused by thermal heating and concentration redistribution is given by

$$
\frac{\mathrm{d} n(r, z)}{\mathrm{d} T}=\left(\frac{\partial n}{\partial T}\right)_{c}+\frac{\partial n}{\partial c} \frac{\partial c}{\partial T}
$$


and in the latter case it is due to transitions of $\mathrm{Fe}_{3} \mathrm{O}_{4} \mathrm{NP}$ electrons to higher energy states by the action of photons with energies higher than the bandgap energy of $\mathrm{Fe}_{3} \mathrm{O}_{4} \mathrm{NP} 0.2 \mathrm{eV}$, which are considered as intraband transitions causing $\Delta n$ [49]. It can be seen from Equation (33) that the effect of $\Delta T(r) \propto R_{p}^{2}$ can consequently cause the change of refractive index hence the beam divergence angle, $\theta_{d}$ i.e., the angle between centered axis of the laser and the diverged beam rays [50] [51].

$$
\theta_{d}=1-(1+2 I)^{1 / 2}
$$

which in this case yields a value of $\theta_{d}=5.24^{\circ} \equiv 91 \mathrm{mrad}$. Applying the values of $\theta_{d}$ and $K_{m}$ in Equation (38)

$$
\frac{\mathrm{d} n}{\mathrm{~d} T}=\frac{\theta_{d} \lambda K_{m}}{P}=1 \times 10^{-5} \mathrm{~K}^{-1} \ll 9 \times 10^{-5} \mathrm{~K}^{-1} \text { for water. }
$$

However, because the NFF concentration used in the experiment is very small one may assume that the initial diffusion coefficient $D_{0}=D(c)$ at a given concentration i.e., it is a concentration independent case. Thus, a step-like variation of concentration in a plane within the medium can be written as

$$
\left(\frac{\partial C}{\partial y}\right)=-\frac{C_{0}}{2 \sqrt{\pi D t}} \mathrm{e}^{\left(-y^{2} / 4 D t\right)}
$$

This is a Gaussian function and has the same shape as the deflected beam trajectory inside the base fluid [52] i.e.,

$$
Z(y)=\Pi \mathrm{e}^{\left(-y^{2} / 4 D t\right)}
$$

where $\Pi$ is a constant. It can be seen from Equation (39) as time elapses, the boundary smears out until the concentration gradient vanishes consequently, the broadening of the Gaussian function occurs. Self-assembly of NPS under influence of electromagnetic field with the frequencies in the optical range has been studied by Park et al. [53] and as suggested by Slabko et al. [54], when NPs are irradiated by the laser radiation, dipole moment is induced which enhances the formation of structural geometry hence forming an agglomeration. In our case, the downward motion of the agglomerates is demonstrated by FITC fluorescence. However, in the case of Brownian dynamics (i.e., no laser), trajectories of an ensemble of NPs in base medium are described by well-known Langevin equation described Equation (3) where the interaction between NPs with environment with fluctuating density results in random change of trajectory movement.

\section{Conclusion}

Dynamics of laser-transport nanoferrofluid was studied by using FITC-conjugated MNPs as marker based on LIF. Based on the Brownian diffusion and DLVO theory, the NPs are more dispersed and free to move within the medium at earlier times. At later stages they become less mobile due to agglomeration. Also, the results showed a laser-induced enhanced velocity of NPs almost twice as much without laser. An initial rapid forward movement was observed when the 
laser was switched on. The measured diffusion coefficients showed a higher value for the case with laser action. The mechanisms for the enhanced mobility and laser transport of NPs are thought to be due to e.m.w induced force (i.e. an oscillatory motion) and laser absorptive force (i.e., photothermophoresis). Also, the laser beam showed a trajectory path due to thermal heating causing the change of refractive index of medium and redistribution of NPs concentration.

\section{References}

[1] Rosenberg, R.E. (1985) Ferrohydrodynamics. Cambridge University Press, Cambridge.

[2] Pankhurst, Q.A., Connolly, J., Jones, S. and Dobson, J. (2003) Journal Physics D: Applied Physics, 36, 167-181. https://doi.org/10.1088/0022-3727/36/13/201

[3] Gupta, A. and Gupta, M. (2005) Biomaterials, 26, 3995-4021. https://doi.org/10.1016/j.biomaterials.2004.10.012

[4] Chou, C., Chen, C. and Wang, C. (2005) Journal Physics Chemistry B, 109, 11135. https://doi.org/10.1021/jp0444520

[5] Jiang, C., McConney, M., Singamaneni, S. and Merrick, E. (2006) Chemistry of Material, 18, 2632-2638. https://doi.org/10.1021/cm060416x

[6] Clement, G., Vincent, D., Veronique, P. and Jerome, F. (2015) Journal Physics Chemistry C, 119, 28148-28154.

[7] Khosroshahi, M.E., Ghazanfari, L., Hasannejad, Z. and Lenhert, S. (2015) Journal of Nanomedicine Nanotechnology, 6, 1-9. https://doi.org/10.4172/2157-7439.1000298

[8] Chertok, B., David, A., Moffat, B. and Yang, V. (2009) Biomaterials, 30, 6780-6787. https://doi.org/10.1016/j.biomaterials.2009.08.040

[9] Mok, H. and Zhang, M. (2013) Expert Opinion Drug Delivery, 10, 73-87. https://doi.org/10.1517/17425247.2013.747507

[10] Khosroshahi, M.E., Alangah, H., Keshvari, H., Bonakdar, Sh. and Tajabadi, M. (2016) Material Science and Engineering C, 62, 544-552. https://doi.org/10.1016/j.msec.2016.01.082

[11] Li, L., Jiang, W., Luo, K., Song, H. and Lan, F. (2013) Theranostics, 3, 595-615. https://doi.org/10.7150/thno.5366

[12] Cao, Y., Jin, R. and Mirkin, C. (2002) Science, 297, 1536-1540. https://doi.org/10.1126/science.297.5586.1536

[13] Ting, B., Zhang, J., Gao, Z. and Yang, J. (2009) Biosensors Bioelectron, 25, 282-287. https://doi.org/10.1016/j.bios.2009.07.005

[14] Bissonnette, L. (1973) Applied Optics, 12, 719-728. https://doi.org/10.1364/AO.12.000719

[15] Phuoc, T., Massoudi, M. and Wang, P. (2016) Fluids, 1, 1-10. https://doi.org/10.3390/fluids1040035

[16] Qian, M., Liu, J., Yan, M. and Shen, Z. (2006) Optics Express, 14, 7559-7566. https://doi.org/10.1364/OE.14.007559

[17] Kleine, H., Gronig, H. and Takayama, K. (2006) Optics and Laser Engineering, 3, 170-189.

[18] Watarai, H., Monjushiro and Tsukahara, S. (2004) Analytical Sciences, 20, 423-434. https://doi.org/10.2116/analsci.20.423

[19] Pu, Sh., Chen, X., Liao, W. and Chen, L. (2004) Journal of Applied Physics, 15, 
5930-5932. https://doi.org/10.1063/1.1808242

[20] Weinert, F. and Braun, D. (2008) Journal of Applied Physics, 104, Article ID: 104701. https://doi.org/10.1063/1.3026526

[21] Fang, X., Yimin, X. and Li, Q. (2009) Applied Laser Physics, 95, Article ID: 203108.

[22] Sutton, J., Fisher, B. and Fleming, J. (2008) Experiments in Fluids, 45, 869-881. https://doi.org/10.1007/s00348-008-0506-4

[23] Khosroshahi, M.E. and Rahmani, M. (2011) Journal Fluorescence, 22, 281-288. https://doi.org/10.1007/s10895-011-0958-4

[24] Qian, J., Li, X., Wei, M. and Gao, X. (2008) Optics Express, 16, 19568-19578. https://doi.org/10.1364/OE.16.019568

[25] Bisker, G., Minai, L. and Yelin, D. (2012) Plasmonics, 7, 609-617. https://doi.org/10.1007/s11468-012-9349-1

[26] Magali, J., Hernandez, A., Maurras, A. and Puget, K. (2009) Tetrahedron Letter, 50, 260-263. https://doi.org/10.1016/j.tetlet.2008.10.141

[27] Rezvani Alanagh, H., Khosroshahi, M.E., Tajabadi, M. and Keshvari, H. (2014) Journal Superconductivity Novel Magnetism, 27, 2337-2345. https://doi.org/10.1007/s10948-014-2598-9

[28] Khosroshahi, M.E., Ghazanfari, L. and Hasannejad, Z. (2017) Journal Nanomedicine Research, 6, 1-10.

[29] Khosroshahi, M.E. and Asemani, M. (2017) International Journal of Nanomaterials, Nanotechnology and Nanomedicine, 3, 44-50.

[30] Jin, M., Shu, H., Liang, P., Cao, D. and Chen, X. (2013) Journal Physical Chemistry C, 117, 23349-23356. https://doi.org/10.1021/jp407520q

[31] Baykal, A., Toprak, M., Durmus, Z., Senel, M., Sozeri, H. and Demir, A. (2012) Journal Superconductivity Novel Magnetism, 25, 1541-1549. https://doi.org/10.1007/s10948-012-1454-Z

[32] Xue, D., Chai, G., Li, X. and Fan, X. (2008) Journal Magnetism Magnetic Materials, 320, 1541-1547. https://doi.org/10.1016/j.jmmm.2008.01.004

[33] Prasher, R., Phelan, P. and Bhattacharya, P. (2006) Nano Letter, 6, 1529-1532. https://doi.org/10.1021/nl060992s

[34] Derjaguin, B. and Landau, L. (1941) Acta Physicochimist URSS, 14, 633.

[35] Verwey, E. and Overbeek, J. (1948) Theory of Stability of Lyophobic Colloids. Elsevier Press, Amsterdam.

[36] Khosroshahi, M.E. and Mandelis, A. (2015) International Journal of Thermophysics, 36, 880-890. https://doi.org/10.1007/s10765-014-1773-3

[37] Khosroshahi, M.E. and Ghazanfari, L. (2010) Physica E, 42, 1824-1829. https://doi.org/10.1016/j.physe.2010.01.042

[38] Zhao, B., Koo, Y. and Chung, D. (2006) Analytical Chemica Acta, 556, 97-103. https://doi.org/10.1016/j.aca.2005.06.065

[39] Liu, X. and Wang, F. (2010) Advances in Optoelectronics and Micro/Nano-Optics, 1-3.

[40] Levitin, E., Kokodiy, N., Timanjuk, V., Vederniova, I. and Chan, T. (2014) Inorganic Materials, 40, 817-820. https://doi.org/10.1134/S0020168514080123

[41] Bost, W., Lemor, R. and Fournelle, M. (2012) Applied Optics, 51, 8041-8046. https://doi.org/10.1364/AO.51.008041

[42] Hossain, M., Kitahama, Y., Huang, G., Han, X. and Ozaki, Y. (2009) Analytical Bioanalytical Chemistry, 394, 1747-1760. 
https://doi.org/10.1007/s00216-009-2762-4

[43] Landu, L. and Lifschits, E. (1987) Fluid Mechanics (Course of Theoretical Physics). Vol. 2, Pergamon Press.

[44] Govorov, A. and Richardson, H. (2007) Nanotoday, 2, 30-38.

[45] Fan, J. and Wang, L. (2011) Journal of Heat Transfer, 133, Article ID: 040801.

[46] Shima, P., Philip, J. and Raj, B. (2010) Journal Physics Chemistry C, 114, 18825-18833. https://doi.org/10.1021/jp107447g

[47] Shima, P. and Philip, J. (2013) Industrial \& Engineering Chemistry Research, 53, 980-988. https://doi.org/10.1021/ie403086g

[48] Delville, J., de Saint Vincent, M., Schroll, R., Chraibi, H. and Issenmann, B. (2009) Journal of Optics A: Pure and Applied Optics, 11, 1-15. https://doi.org/10.1088/1464-4258/11/3/034015

[49] Milichko, V., Nechaev, A., Valtsifer, V. and Strelnikov, V. (2013) Nanoscale Research Letters, 8, 317-324. https://doi.org/10.1186/1556-276X-8-317

[50] Kurian, A., Kumar, R. and George, S. (2009) Proceedings of SPIE, 7393, 73930U. https://doi.org/10.1117/12.826233

[51] Koyanaka, Sh. and Endoh, Sh. (1999) Advanced Powder Technology, 10, 205-221. https://doi.org/10.1163/156855299X00307

[52] Kurian, A., Bindhu, C., Harilal, S., Issac, R., Nampoori, V. and Vallabhan, C. (1994) Pramana Journal of Physics, 43, 401-406.

[53] Park, J. and Lu, W. (2011) Journal Physics Review E, 83, Article ID: 031402.

[54] Slabko, V., Tsipotan, A., Aleksandrovsky, A. and Slyuareva, E. (2014) Applied Physics B, 117, 271-278. https://doi.org/10.1007/s00340-014-5831-0 